Biological and Clinical Sciences Research Journal

ISSN: 2708-2261

www.bcsrj.com

DOI: https://doi.org/10.54112/bcsrj.v2020i1.40

Biol. Clin. Sci. Res. J., Volume, 2020: 40

Original Research

\title{
DIAGNOSTIC ACCURACY OF ULTRASOUND IN DETECTING MENISCAL TEARS TAKING MAGNETIC RESONANCE IMAGING AS GOLD STANDARD
}

\author{
OMER MA ${ }^{1^{*}}$, MALIK SS ${ }^{2}$, MALIK AA ${ }^{3}$, ANJUM MN ${ }^{2}$, RIAZ AZ ${ }^{4}$, ALI R \\ ${ }^{l}$ Department of Diagnostic Radiology, The University of Lahore, Lahore, Pakistan \\ ${ }^{2}$ Radiology Research Section, The University of Lahore, Lahore, Pakistan \\ ${ }^{3}$ Consultant Interventional Radiologist, Doctors Hospital and Medical Center, Lahore, Pakistan \\ ${ }^{4}$ House Officer Radiology, Services Hospital Lahore, Lahore, Pakistan \\ ${ }^{5}$ Consultant Radiologist DHQ Hospital Sheikupura, Pakistan \\ Corresponding \& First Author email: dr.arslan14@gmail.com
}

(Received, $5^{\text {th }}$ July 2020, Revised $10^{\text {th }}$ November 2020, Published $18^{\text {th }}$ November 2020)

\begin{abstract}
Meniscal tears are mostly seen worldwide. Multiple imaging modalities are currently used to evaluate pathologic conditions of the knee. MRI can be referred as the non-invasive gold standard investigation to help establishing diagnosis of meniscal tears, but it economically unaffordable to most of patients and needs long examination times. Ultrasound is a possible alternative to MRI. It is simple, convenient and an inexpensive and noninvasive method. The use of ultrasound in identifying meniscal tears has been proposed, but its diagnostic accuracy remains controversial. The objective of the research is to define the diagnostic accurateness of ultrasound scan in detecting meniscal tears taking magnetic resonance imaging as gold standard. It was a diagnostic cross-sectional study in which 78 patients visiting Department of Diagnostic Radiology, Doctors Hospital, Lahore were included. Convenient sampling technique was used. Written informed consent was taken from all the patients. Patients were investigated with the help of ultrasound and results were noted on the designed proforma, subsequently the results were compared to the MRI scan of the knee joint. Data was entered and statistically analyzed using SPSS 21.0. Frequency tables were generated for all variables. For quantitative data like age mean and standard deviation were calculated and for qualitative data like sex and meniscal tears percentages were calculated. Sensitivity, Specificity, Positive Predictive Value, Negative Predictive Value was given. Bar and pie charts were used to present categorical data. Kappa analysis was utilized. The mean age of the patients was $38.18+12.818$ years, $82.1 \%$ were males and $47.4 \%$ had sports trauma. Out of 48 medial meniscus tear on USG, 38(48.7\%) were true positive and 10(12.8\%) were false positive on MRI. Out of 10 lateral meniscus tear on USG, 9(11.5\%) were true positive and 1(1.3\%) was false positive on MRI. The sensitivity, specificity, PPV, NPV and diagnostic accuracy of ultrasound for medial meniscus tears were $95.00 \%, 73.68 \%, 79.16 \%, 93.33 \%$ and $84.61 \%$ while for lateral meniscus tears were $75.00 \%$, $98.48 \%, 90.00 \%, 95.58 \%$ and $94.87 \%$, respectively. Study concluded that ultrasound has good diagnostic accuracy when compared with magnetic resonance imaging in detecting meniscal tears.
\end{abstract}

Keywords: Ultrasound, meniscal tears, magnetic resonance imaging, diagnosis, sensitivity

increases contact area and hence decreases contact

\section{Introduction}

The knee joint is one of the most vulnerable joint of the body (Sharma and Grewal, 2020). The ligaments establish the chief supportive structure of the knee joint. Owing to the fact that the bony structures of knee joint is restricted, the other structure including ligaments, tendons, menisci and cartilages makes the main role to stabilize the knee joint and prevent it from injuries (Sally and Ali, 2019). Menisci are vital for the functional well-being of the knee. An intact meniscus is crucial for maintaining normal knee function, such as shock absorption, joint lubrication and load transmission across the knee joint. It stress on the articular cartilage (Stewart, 2010). The most common causes of knee pain and disability are tears in medial or lateral menisci (Attya, 2015). Meniscal tears are mostly seen worldwide (Snoeker et al., 2013) and are common in both athletes and non-athletes. They can cause knee pain and disability as well as the onset and progression of knee osteoarthritis (Akatsu et al., 2015). The prevalence of asymptomatic tears, which typically are horizontal tears, increases with age (Nguyen et al., 2014). Meniscal tears are the most widely recognized pathology of the knee with a mean yearly occurrence of 66 for every 100000 (Mostafa et al., 2019). Almost two third of patients of older age (more 65

[Citation: Omer, M.A., Malik, S.S., Malik, A.A., Anjum, MN., Riaz, A.Z., Ali, R. (2020). Diagnostic accuracy of ultrasound in detecting meniscal tears taking magnetic resonance imaging as gold standard. Biol. Clin. Sci. Res. J., 2020: 40. doi: https://doi.org/10.54112/bcsrj.v2020i1.40] 
years) experience progressive meniscal tears. Situated in between the tibial and femoral bones, two semilunar segments structure of knee joint called the menisci (Mahdy et al., 2018). The medial meniscus is C-shaped structure measuring about $3.5 \mathrm{~cm}$ longitudinally beginning anteriorly towards posteriorly (Acebes et al., 2013). It is unequal with a significantly broader posterior end than frontal end. The lateral meniscus is almost round shape making a circle covering most of the area of tibial upper surface (Najafi et al., 2006; Razek et al., 2009). The medial meniscus by its center portion establishes secure links with fibers of the collateral ligaments (through its deep medial part) (Nogueira-Barbosa et al., 2015).

Meniscal tears has 6 subtypes: 1. Longitudinal tears; makes bucket shape when dislocated, 2. Radial tears, 3. oblique flap (Parrot-beak) tears, 4. Horizontal tears, 5. Root tears, 6. A mixture of all the above subtypes called the Complex tears. The classification of meniscal tears provides a description of pathoanatomy. Warner and Harner classified meniscal tears on the basis of their location in three zones of vascularity - red-red (fully within the vascular area), red-white (at the border of the vascular area), and white (within the avascular area). They use this classification to determine the potential for healing after repair. Tears in red-red and redwhite zones have good potential for healing after repair. Successful repair and healing relieves meniscal symptoms and allows the patient to return to full function. Multiple imaging modalities are currently used to evaluate pathologic conditions of the knee (Murmu et al., 2017). Clinical diagnosis of meniscal tears can be difficult even for the most experienced orthopedic surgeons, which is most often diagnosed based on history, clinical symptoms, magnetic resonance imaging (MRI), or arthroscopy. Arthroscopy is main diagnostic standard test, but its negative aspects include that it is very costly, invasive procedure and necessitate hospital stay for surgery (Dong et al., 2018).

Magnetic resonance imaging has attained acceptance as an analytical means for the musculoskeletal system since its introduction in the 1980s (Wang et al., 2019). Magnetic resonance imaging is not invasive and does not require exposure to ionizing radiation (Alizadeh et al., 2013). Since then, due to the better signal-to-noise ratio, higher resolution, reduced number of artifacts, shorter imaging time and better accuracy, MRI has clearly become the main imaging tool in knee joint pathology studies (Phelan et al., 2016; Wang et al., 2019). MRI not only depicts osseous lesions, but provides information on the cartilages, menisci, ligaments and surrounding softtissues. On MRI meniscal tears are characterized by linear, complex, or diffuse increased signal intensity within the meniscus which communicates with an articular surface (Arif et al., 2013). It gives a high sensitivity of $93 \%$ for medial meniscus and $79 \%$ for lateral meniscus and also a high specificity of $88 \%$ for medial meniscus and $96 \%$ for lateral meniscus for diagnosing the meniscal tears (Choi et al., 2010). MRI can be taken as the non-invasive "diagnostic gold standard" for meniscal tears, its high cost and the long examination times are the negative points (De Smet et al., 2009). Diagnostic ultrasound of the knee can identify abnormalities in the menisci. It is simple, convenient and an inexpensive and noninvasive method (Oei et al., 2003). Ultrasound can accurately determine the location and presence of tear. It also can differentiate complete tears from partial tears, an important distinction because the former requires surgery. Partial tears are seen as a cleft or anechoic region with the tendon with interruption of its normal echogenic fibrillar pattern. Complete tears are seen as complete disruption of the tendon with separated ends (Lee and Chow, 2007). The use of ultrasound (US) as a clinical investigative tool started in 1950's. US application in imaging remained underutilized until 1980's. It is a noninvasive, freely available, well-accepted by patients, affordable and dynamic evaluation in real time. Recent advances in US system instrumentation and transducer technology allowed better demonstration of musculoskeletal diseases. The most known clinical application is the ability to obtain a clear anatomical overview of the superficial structures around the bones. Soft tissue pathology of the knee represented one of the common uses since the nineties of the last century (Yousif et al., 2014). Ultrasound is a possible alternative to MRI and can be performed rapidly, is less costly, and can be used to evaluate muscles and tendons. Although the depth of penetration of ultrasound is limited, recent developments in ultrasound have led to greater spatial resolution as well as finer imaging detail than are possible with standard clinical musculoskeletal MRI; therefore, its usefulness for diagnosing meniscal disease seems promising. The use of ultrasound for identifying meniscal tears has been proposed, but its diagnostic accuracy remains controversial (Dai et al., 2015). Problems with previous studies of ultrasound include a failure to address the resolution of the machine. Therefore, the current research aims to evaluate the diagnostic correctness of ultrasound scanning for detecting meniscal tears taking Magnetic Resonance Imaging as Gold Standard.

[Citation: Omer, M.A., Malik, S.S., Malik, A.A., Anjum, MN., Riaz, A.Z., Ali, R. (2020). Diagnostic accuracy of ultrasound in detecting meniscal tears taking magnetic resonance imaging as gold standard. Biol. Clin. Sci. Res. J., 2020: 40. doi: https://doi.org/10.54112/bcsrj.v2020i1.40] 
Material and methods

Study design

Diagnostic cross-sectional

Study setting

Department of Diagnostic Radiology, Doctors

Hospital, Lahore

Study duration

9 months.

Sample size

78 patients according to following formula:

Population size (for finite population correction factor or $\mathrm{fpc})(\mathrm{N}): 1000000$

Hypothesized \% frequency of outcome factor in population (p): $86.4 \%+/-5$

Confidence limits \% of 100 (absolute +/- \%)(d): 5\%

Design effects (for cluster surveys-DEFF): 1

\section{Equation}

Sample Size $n=[\operatorname{DEFF} \times \mathbf{N p}(\mathbf{1}-\mathbf{p})] /\left[\left(\mathbf{d}^{2} / \mathbf{Z}_{1-\mathrm{a} / 2}^{2} \times(\mathbf{N}-\right.\right.$

1) $+\mathbf{p} \times(\mathbf{1 - p})]$

Sampling technique

Convenient sampling

Sample selection

Inclusion Criteria

Patients between the age of 15 to 60 years of both genders with knee pain \& positive in at minimum any of the following bedside examination:

- Joint Line Tenderness

- $\quad$ McMurray Test

- $\quad$ Apley Grind Test

Exclusion Criteria

- $\quad$ Unfit for MRI Investigation.

- $\quad$ History of prior knee surgery.

- Unwilling to participate.

\section{Instruments/modality}

Canon/Toshiba Aplio 500 Platinum with Wideranging Group In lines transducer with rate of recurrence of 7-18 Mhz.

\section{Ethical considerations}

- Written informed consent was taken from all the patients.

- All information and collected data was kept confidential by saving data in my personal laptop and hard copies form data were in locker.

- Contributors stayed unnamed during the course of the research.

- It was brought to the knowledge of the participants; they will have no difficulties or risk from the procedures of the study.

\section{Results}

Table 1 describes that among 78 patients, 26 (33.3\%) were upto 30 years of age, 19 (24.3\%) were $31-40$ years of age and $17(22.0 \%)$ were 41-50 years of age while $16(20.4 \%)$ cases were more than 50 years of
- They were similarly acknowledged that they will be free to remove themselves at any time throughout the course of the study.

- Printed record was stored in the lockers and soft copy was password protected in the laptop.

\section{Data collection procedure}

A Performa was planned by researcher and decided after pre-testing. Those patients who visited Doctors' Hospital \& Medical Center, Lahore were included and investigated with the help of ultrasound and results were noted on the designed Performa, subsequently the results were compared to the MRI scan of the knee joint.

Technique and equipment: The ultrasonography examination was done with help of Canon/Toshiba Aplio 500 Platinum with Wide-ranging Group In lines transducer with rate of recurrence of 7-18 Mhz.

Patients positioning: each participant was inspected in supine laying position with knee joint flexed (at $20^{\circ}-30^{\circ}$ ) for evaluation of anterior side of the knee, while the posterior side was inspected in prone situation with the knee not flexed (i.e. straight) and the cruciate ligament flexed (at $60^{\circ}-70^{\circ}$ ).

Medial collateral ligament and medial meniscus: The leg was turned externally with flexion slightly (at $20^{\circ}-30^{\circ}$ ) for the inspection of the medial side of the knee joint. Heavy part (which constitute hyperechoic fibrillar outer layer) and thinner part (which constitute the deeper layer) are the two components which make the medial collateral ligament. The hyperechoic wedge structure that lies between the femur and tibia is refered as the medial meniscus.

Lateral collateral ligament and lateral meniscus: the leg was turned internally with slight knee flexion $\left(\right.$ at $20^{\circ}-30^{\circ}$ ) for the lateral side inspection of the knee joint. Also the examination of lateral collateral ligament and the anterior horn of lateral ligament are possible at this position.

\section{Data analysis}

Data was entered \& analyzed using SPSS (Statistical Package for Social Sciences) version 22.0. Frequency tables were generated for all variables. For quantitative data like age and, mean and standard deviation were calculated and for qualitative data like sex and meniscal tears percentages were calculated. Sensitivity, Specificity, Positive Predictive Value, Negative Predictive Value was given. Bar and pie charts were used to present categorical data. Kappa analysis was utilized.

age. The mean age of the individuals was $38.18 \pm 12.818$ years. Table 1 depicts that among 78 patients, $64(82.1 \%)$ were males and only $14(17.9 \%)$ were females.

[Citation: Omer, M.A., Malik, S.S., Malik, A.A., Anjum, MN., Riaz, A.Z., Ali, R. (2020). Diagnostic accuracy of ultrasound in detecting meniscal tears taking magnetic resonance imaging as gold standard. Biol. Clin. Sci. Res. J., 2020: 40. doi: https://doi.org/10.54112/bcsrj.v2020i1.40] 
Table 1. Frequency distribution of patients according to age and gender

\begin{tabular}{lll}
\hline Age & Frequency & Percentage (\%) \\
\hline Upto 30 years & 26 & 33.3 \\
31-40 years & 19 & 24.3 \\
41-50 years & 17 & 22.0 \\
Above 50 years & 16 & 20.4 \\
Total & 78 & 100.0 \\
Mean+SD & $38.18 \pm 12.818$ & \\
Sex & Frequency & Percentage (\%) \\
\hline Male & 64 & 82.1 \\
Female & 14 & 17.9 \\
Total & 78 & 100.0 \\
\hline \multicolumn{1}{c}{ Table 2. Occurrence distribution of patients depending } & on & mode of damages \\
\hline Mode of Injury & Frequency & Percentage (\%) \\
\hline Road Accident & 5 & 6.4 \\
Senile Changes & 2 & 2.6 \\
Sports Trauma & 37 & 47.4 \\
Control as per MRI & 34 & 43.6 \\
Total & 78 & 100.0 \\
Knee side & Frequency & Percentage (\%) \\
\hline Right & 58 & 74.4 \\
Left & 16 & 20.5 \\
Both & 4 & 5.1 \\
Total & 78 & 100.0 \\
Medial meniscus tears on USG & Frequency & Percentage (\%) \\
\hline Anterior Zone Tear & 9 & 11.5 \\
Middle Zone Tear & 6 & 7.7 \\
Posterior Zone Tear & 33 & 42.3 \\
No Tear & 30 & 38.5 \\
Total & 78 & 100.0 \\
Lateral meniscus tears on USG & Frequency & Percentage (\%) \\
\hline Anterior Zone Tear & 0 & 0.0 \\
Middle Zone Tear & 3 & 3.8 \\
Posterior Zone Tear & 7 & 9.0 \\
Normal & 68 & 87.2 \\
Total & 78 & 100.0 \\
\hline & 18 & $1 \%$ \\
\hline
\end{tabular}

Above table 2 demonstrates the mode of injury among patients and found that $5(6.4 \%)$ patients had road accident, $2(2.6 \%)$ senile changes and majority $37(47.4 \%)$ had sports trauma while 34 (43.6\%) were control as per MRI. Table 2 shows that out of 78 patients, right knee of $58(74.4 \%)$ patients was examined and left knee of $16(20.5 \%)$ patients was examined while both knees of $4(5.1 \%)$ patients were examined. Table 2 exhibits that according to USG findings, $9(11.5 \%)$ patients had medial meniscus tears in anterior zone, $6(7.7 \%)$ patients in middle zone, $33(42.3 \%)$ in posterior zone and $30(38.5 \%)$ patients had no tear. Table 2 indicates that according to USG findings, none of the patients had lateral meniscus tears in anterior zone, $3(3.8 \%)$ patients had in middle zone and $7(9.0 \%)$ in posterior zone while majority of the patients had no tear $68(87.2 \%)$. Table 3 asserts that according to MRI findings, 8 (10.3\%) patients had medial meniscus tears in anterior zone, 3 $(3.8 \%)$ patients in middle zone, 29 (37.2\%) in posterior zone and $38(48.7 \%)$ patients had no tear. Table 3 highlights that according to MRI findings, none of the patients had lateral meniscus tears in anterior zone, $3(3.8 \%)$ patients had in middle zone and $9(11.5 \%)$ in posterior zone while majority of the patients had no tear $66(84.7 \%)$.

Table 3. Frequency distribution of patients according to medial meniscus tears on MRI

\begin{tabular}{lll}
\hline Medial Meniscus tears on MRI & Frequency & Percentage (\%) \\
\hline Anterior Zone Tear & 8 & 10.3 \\
Middle Zone Tear & 3 & 3.8 \\
Posterior Zone Tear & 29 & 37.2 \\
\hline
\end{tabular}

[Citation: Omer, M.A., Malik, S.S., Malik, A.A., Anjum, MN., Riaz, A.Z., Ali, R. (2020). Diagnostic accuracy of ultrasound in detecting meniscal tears taking magnetic resonance imaging as gold standard. Biol. Clin. Sci. Res. J., 2020: 40. doi: https://doi.org/10.54112/bcsrj.v2020i1.40] 


\begin{tabular}{lll}
\hline Normal & 38 & 48.7 \\
Total & 78 & 100.0 \\
Lateral meniscus tears on MRI & Frequency & Percentage (\%) \\
\hline Anterior Zone Tear & 0 & 0.0 \\
Middle Zone Tear & 3 & 3.8 \\
Posterior Zone Tear & 9 & 11.5 \\
Normal & 66 & 84.7 \\
Total & 78 & 100.0 \\
\hline
\end{tabular}

Table 4 reveals that out of 58 meniscus tears on USG, 48 were medial tears and 10 were lateral tears. Out of 48 medial tears, $9(11.5 \%)$ were found in anterior zone, $6(7.7 \%)$ in middle zone and $33(42.3 \%)$ in posterior zone. Likewise out of 10 lateral tears, 3 (3.8\%) were found in middle zone and $7(9.0 \%)$ in posterior zone. Table 5 indicates that out of 52 meniscus tears on MRI, 40 were medial tears and 12 were lateral tears. Out of 40 medial tears, $8(10.3 \%)$ were found in anterior zone, $3(3.8 \%)$ in middle zone and $29(37.2 \%)$ in posterior zone. Likewise out of 12 lateral tears, $3(3.8 \%)$ were found in middle zone and $9(11.5 \%)$ in posterior zone. Table 6 depicts that out of 48 medial meniscus tear on USG, 38 (48.7\%) were true positive and $10(12.8 \%)$ were false positive on
MRI. Out of 30 medial meniscus without tear, $2(2.6 \%)$ were false negative and $28(35.9 \%)$ were true negative on MRI. The Kappa value was 0.000 . Table 7 demonstrates that out of 10 lateral meniscus tear on USG, $9(11.5 \%)$ were true positive and 1 (1.3\%) was false positive on MRI. Out of 68 lateral meniscus without tear, $3(3.8 \%)$ were false negative and $65(83.4 \%)$ were true negative on MRI. The Kappa value was 0.000 . Table 8 depicts that sensitivity, specificity, PPV, NPV and diagnostic accuracy of ultrasound for medial meniscus tears were $95.00 \%, 73.68 \%, 79.16 \%, 93.33 \%$ and $84.61 \%$ while for lateral meniscus tears were $75.00 \%$, $98.48 \%, 90.00 \%, 95.58 \%$ and $94.87 \%$, respectively.

Table 4. Breakdown of meniscus tears on USG

\begin{tabular}{llll}
\hline & Anterior Zone & Middle Zone & Posterior Zone \\
\hline Medial meniscus & $9(11.5 \%)$ & $6(7.7 \%)$ & $33(42.3 \%)$ \\
Lateral meniscus & $0(0.0 \%)$ & $3(3.8 \%)$ & $7(9.0 \%)$ \\
\hline
\end{tabular}

Total Meniscus Tear: 58, Medial Tear 48, Lateral Tear: 10

Table 5. Breakdown of meniscus tears on MRI

\begin{tabular}{llll}
\hline & Anterior Zone & Middle Zone & Posterior Zone \\
\hline Medial meniscus & $8(10.3 \%)$ & $3(3.8 \%)$ & $29(37.2 \%)$ \\
Lateral meniscus & $0(0.0 \%)$ & $3(3.8 \%)$ & $9(11.5 \%)$ \\
\hline
\end{tabular}

Total Meniscus Tear: 52, Medial Tear 40, Lateral Tear: 12

Table 6. Diagnosis of medial meniscus tears by ultrasound and MRI

\begin{tabular}{llll}
\hline Ultrasound findings & $\begin{array}{l}\text { MRI findings } \\
\text { Meniscus with tear }\end{array}$ & Meniscus without tear & \multirow{2}{*}{ Total } \\
\hline Meniscus with tear & $38(48.7 \%)$ & $10(12.8 \%)$ & $48(61.5 \%)$ \\
Meniscus without tear & $2(2.6 \%)$ & $28(35.9 \%)$ & $30(38.5 \%)$ \\
Total & $40(51.3 \%)$ & $38(48.7 \%)$ & $78(100.0 \%)$ \\
\hline
\end{tabular}

Kappa $=0.000$

Table 7. Diagnosis of lateral meniscus tears by ultrasound and MRI

\begin{tabular}{llll}
\hline \multirow{2}{*}{ Ultrasound findings } & $\begin{array}{l}\text { MRI findings } \\
\text { Meniscus with tear }\end{array}$ & Meniscus without tear & Total \\
\hline Meniscus with tear & $9(11.5 \%)$ & $1(1.3 \%)$ & $10(12.8 \%)$ \\
Meniscus without tear & $3(3.8 \%)$ & $65(83.4 \%)$ & $68(87.2 \%)$ \\
Total & $12(15.3 \%)$ & $66(84.7 \%)$ & $78(100.0 \%)$ \\
\hline
\end{tabular}

Kappa $=0.000$

Table 8. Diagnostic accurateness of ultrasound in detecting meniscus tears

\begin{tabular}{lll}
\hline & Medial meniscus & Lateral meniscus \\
\hline Sensitivity & $95.00 \%$ & $75.00 \%$ \\
Specificity & $73.68 \%$ & $98.48 \%$ \\
Positive predictive value (PPV) & $79.16 \%$ & $90.00 \%$ \\
Negative predictive value (NPV) & $93.33 \%$ & $95.58 \%$ \\
Diagnostic accuracy (DA) & $84.61 \%$ & $94.87 \%$ \\
\hline
\end{tabular}

[Citation: Omer, M.A., Malik, S.S., Malik, A.A., Anjum, MN., Riaz, A.Z., Ali, R. (2020). Diagnostic accuracy of ultrasound in detecting meniscal tears taking magnetic resonance imaging as gold standard. Biol. Clin. Sci. Res. J., 2020: 40. doi: https://doi.org/10.54112/bcsrj.v2020i1.40] 


\section{DISCUSSION}

Menisci are significant for the functional well being of the knee. An undamaged meniscus is important for maintaining the normal function of knee namely joint lubrication, shock absorption, and weight transmission across the knee joint. For knee pain and disability, the most frequent reasons are tears in lateral or medial menisci. The meniscal tears can lead to knee pain, disability and onset \& knee osteoarthritis progression. Several imaging modalities are presently utilized to assess pathologic conditions of knee. Clinical diagnosis of meniscal tears can be difficult even for the most experienced orthopedic surgeons, which is most often diagnosed based on history, clinical symptoms, magnetic resonance imaging or arthroscopy. MRI not only depicts osseous lesions, but provides information on the cartilages, menisci, ligaments and surrounding softtissues. MRI can be considered as the non-invasive "gold standard" for the diagnosis of meniscal tears, but it is expensive and needs long examination times. Diagnostic ultrasound of the knee can identify abnormalities in the menisci. Ultrasound can accurately determine the location and presence of tear. It is simple, noninvasive, freely available, wellaccepted by patients, inexpensive and dynamic evaluation in real time. Therefore, current study is carried out to assess the diagnostic accuracy of ultrasound scanning in detecting meniscal tears taking magnetic resonance imaging as gold standard. To acquire adequate outcomes, a group of 78 patients was included in the study and found that only $33.3 \%$ patients were up to 30 years old and majority (66.7\%) was more than 50 years old while the mean age of the patients was $38.18 \pm 12.818$ years. But a study carried out by Shetty (2013) reported that majority of the patients (58.4\%) were upto 30 years old and $41.6 \%$ were more than 30 years old while the mean age of the patients was $30.66 \pm 10.58$ years. The findings of our study are comparable with a recent study undertaken by Mostafa and his coworkers (2019) who asserted that mean age of the patients was 37.65 \pm 10.24 . Another study performed by Razek and his associates indicated that mean age of the patients was $41.2 \pm 11.5$ years (Razek et al., 2009). It has been confirmed that mean age of the patients was $30.42 \pm 5.91$ (Shetty, 2013). As far as gender of the patients is concerned, study revealed that disease was more prevalent among male than female patients. It was found that significant majority $(82.1 \%)$ of the patients were male and only $17.9 \%$ were female patients. Several studies carried out in different parts of the worlds also showed similar situation. In a study Yousif et al., (2014) reported that most of the patients $(83.0 \%)$ were male and only $17.0 \%$ were female patients. Similarly, Sharma and Grewal (2020) highlighted in their study that $65.0 \%$ patients were male and $35.0 \%$ were female. Another study conducted by Mostafa et al., (2019) elucidated that $62.0 \%$ were male and $38.0 \%$ were female patients. When the mode of injury was assessed among patients, study disclosed that most of the patients (47.4\%) had sports trauma, followed by road accident $(6.4 \%)$ and senile changes $(2.6 \%)$ while $43.6 \%$ were control as per MRI. The results of a similar study undertaken by Murmu and his fellows reported that majority of the patients (70.59\%) had sports trauma, followed by domestic falls $(11.76 \%)$ and road accident (5.89\%) while $11.76 \%$ cases had miscellaneous causes (Murmu et al., 2017).

Study revealed that among $74.4 \%$ patients, right knee was affected and in $20.5 \%$ patients, left knee was affected while in $5.1 \%$ patients both knees were affected. While a study done by Razek and his associates indicated that among $67.0 \%$ patients; left knee was affected while among $33 \%$ patients, right knee was affected (Razek et al., 2009). Likewise, a study carried out by Arif and his comrades (2013) also confirmed that among majority (72.0\%) of patients; left knee was affected while among $28.0 \%$ patients, right knee was affected. But a study done by Murmu and his fellows showed almost comparable results that among $62.75 \%$ patients, right knee was affected and in $37.25 \%$ patients, left knee was affected (Murmu et al., 2017). It was found during study that according to USG findings, medial meniscus tears (anterior, middle and posterior zone tears) were detected in $61.5 \%$ patients and $38.5 \%$ patients had no tear. But lateral meniscus tears (anterior, middle and posterior zone tears) were detected in only $12.8 \%$ patients and majority $(87.2 \%)$ of the patients had no tear. In another similar study Yaseen and Gorial asserted that as per ultrasound results, meniscal tear were detected among 70\% patients while $30.0 \%$ patients had no tear (Yaseen and Gorial, 2019). Study further disclosed as per MRI findings, medial meniscus tears (anterior, middle and posterior zone tears) were detected among $51.3 \%$ patients while $48.7 \%$ patients had no tear. But lateral meniscus tears (middle and posterior zone tears) were detected in only $15.3 \%$ patients and major proportion (84.7\%) of the patients had no tear. While study carried out by Yaseen and Gorial (2019) reported that according to MRI findings, medial meniscal tears were identified among $88 \%$ patients while $12 \%$ patients had no tear (Yaseen and Gorial, 2019). The outcomes of study indicated that

[Citation: Omer, M.A., Malik, S.S., Malik, A.A., Anjum, MN., Riaz, A.Z., Ali, R. (2020). Diagnostic accuracy of ultrasound in detecting meniscal tears taking magnetic resonance imaging as gold standard. Biol. Clin. Sci. Res. J., 2020: 40. doi: https://doi.org/10.54112/bcsrj.v2020i1.40] 
according to USG, 48 were medial meniscus tears and 10 were lateral meniscus tears. Out of 48 medial meniscus tears, 9 were found in anterior zone, 6 in middle zone and 33 in posterior zone. Likewise out of 10 lateral tears, 3 were found in middle zone and 7 in posterior zone. But according to MRI findings, 40 were medial meniscus tears and 12 were lateral meniscus tears. Out of 40 medial meniscus tears, 8 were found in anterior zone, 3 in middle zone and 29 in posterior zone. Similarly out of 12 lateral tears, 3 were found in middle zone and 9 in posterior zone. A study carried out by Gadgil and his partners (2018) indicated that most of the patients had medial meniscal tear (123), followed by lateral meniscal tear (25) and both medial \& lateral meniscu tear (12) (Gadgil et al., 2018).

The results of our study further indicated that out of 48 medial meniscus tear on USG, 38 were true positive and 10 were false positive on MRI. Out of 30 medial meniscus without tear on USG, 2 were false negative and 28 were true negative on MRI. Similarly out of 10 lateral meniscus tear on USG, 9 were true positive and 1 was false positive on MRI. Out of 68 lateral meniscus without tear on USG, 3 were false negative and 65 were true negative on MRI. A study indicated that out of 42 meniscus tear on USG, 41 were true positive and 1 was false positive on MRI. Out of 17 meniscus without tear on USG, 7 were false negative and 10 were true negative on MRI (Crawford et al., 2007). The findings of another study undertaken by Mostafa and his coworkers reported that out of 26 meniscus tear on USG, 16 were true positive and 10 were false positive on MRI. Out of 36 meniscus without tear on USG, 2 were false negative and 32 were true negative on MRI (Mostafa et al., 2019).

It was very encouraging that sensitivity, specificity, PPV, NPV and diagnostic accuracy of ultrasound for medial meniscus tears were $95.00 \%, 73.68 \%$, $79.16 \%, 93.33 \%$ and $84.61 \%$ while for lateral meniscus tears were $75.00 \%, 98.48 \%, 90.00 \%$, $95.58 \%$ and $94.87 \%$, correspondingly. The findings of our research demonstrated more useful results than the finding of previous researchers where the specificity, sensitivity, PPV, NPV and diagnostic accuracy of ultrasound for medial meniscus tears were $80.7 \%, 62.5 \%, 75.0 \%, 70.0 \%$ and $72.0 \%$ while for lateral meniscus tears were $23.0 \%, 89.1 \%, 42.8 \%$, $76.7 \%$ and $72.0 \%$, respectively (Bien et al., 2018; Cimino et al., 2010; Micheo et al., 2010). It has been reported that assuming the magnetic resonance imaging as gold standard, the sensitivity, specificity, PPV, NPV and diagnostic accuracy of ultrasound were $85.4 \%, 90.9 \%, 97.6 \%, 58.8 \%$ and $86.4 \%$, respectively (Crawford et al., 2007). Another study performed by Alizadeh et al., (2013) highlighted that sensitivity, specificity, PPV, NPV and DA of ultrasound in detecting meniscal tears were $100.0 \%$, $88.9 .0 \%, 96.5 \%, 100.0 \%$ and $97.3 \%$, respectively.

\section{Conclusion}

Meniscal tears are most common public health problems worldwide and can lead to knee pain, disability and knee osteoarthritis. Present study assessed the diagnostic accuracy of ultrasound scanning in detecting meniscal tears taking magnetic resonance imaging as gold standard. Study concluded that ultrasound has good diagnostic accuracy when compared with magnetic resonance imaging in detecting meniscal tears. It is comparatively simple and inexpensive investigation and could be utilized in the clinical practice. Further studies are need on large scale to assess the diagnostic accuracy of ultrasound scanning in detecting meniscal tears.

\section{Conflict of interest}

The authors declared absence of any conflict of interest for manuscript publication.

\section{References}

Acebes, C., Romero, F. I., Contreras, M. A., Mahillo, I., and Herrero-Beaumont, G. (2013). Dynamic ultrasound assessment of medial meniscal subluxation in knee osteoarthritis. Rheumatology 52, 1443-1447.

Akatsu, Y., Yamaguchi, S., Mukoyama, S., Morikawa, T., Yamaguchi, T., Tsuchiya, K., Iwasaki, J., Akagi, R., Muramatsu, Y., and Katsuragi, J. (2015). Accuracy of highresolution ultrasound in the detection of meniscal tears and determination of the visible area of menisci. JBJS 97, 799-806.

Alizadeh, A., Jandaghi, A. B., Zirak, A. K., Karimi, A., Mardani-Kivi, M., and Rajabzadeh, A. (2013). Knee sonography as a diagnostic test for medial meniscal tears in young patients. European Journal of Orthopaedic Surgery \& Traumatology 23, 927-931.

Arif, U., Shah, Z. A., Khan, M. A., Ijaz, M., and Qayum, H. (2013). Diagnostic accuracy of 1.5 tesla MRI in the diagnosis of meniscal tears of knee joint. Arthroscopy 42, 25.

Attya, M. S. (2015). Evaluation of role of non ionized radiology tools in knee soft tissue injuries. AlAzhar Assiut Medical Journal 13, 1.

Bien, N., Rajpurkar, P., Ball, R. L., Irvin, J., Park, A., Jones, E., Bereket, M., Patel, B. N., Yeom, K. W., and Shpanskaya, K. (2018). Deeplearning-assisted diagnosis for knee magnetic resonance imaging: development and

[Citation: Omer, M.A., Malik, S.S., Malik, A.A., Anjum, MN., Riaz, A.Z., Ali, R. (2020). Diagnostic accuracy of ultrasound in detecting meniscal tears taking magnetic resonance imaging as gold standard. Biol. Clin. Sci. Res. J., 2020: 40. doi: https://doi.org/10.54112/bcsrj.v2020i1.40] 
retrospective validation of MRNet. PLoS medicine 15, e1002699.

Choi, C.-J., Choi, Y.-J., Lee, J.-J., and Choi, C.-H. (2010). Magnetic resonance imaging evidence of meniscal extrusion in medial meniscus posterior root tear. Arthroscopy: The Journal of Arthroscopic \& Related Surgery 26, 16021606.

Cimino, F., Volk, B. S., and Setter, D. (2010). Anterior cruciate ligament injury: diagnosis, management, and prevention. American family physician 82, 917-922.

Crawford, R., Walley, G., Bridgman, S., and Maffulli, N. (2007). Magnetic resonance imaging versus arthroscopy in the diagnosis of knee pathology, concentrating on meniscal lesions and ACL tears: a systematic review. British medical bulletin 84, 5-23.

Dai, H., Huang, Z.-g., Chen, Z.-j., and Liu, J.-x. (2015). Diagnostic accuracy of ultrasonography in assessing meniscal injury: meta-analysis of prospective studies. Journal of Orthopaedic Science 20, 675-681.

De Smet, A. A., Blankenbaker, D. G., Kijowski, R., Graf, B. K., and Shinki, K. (2009). MR diagnosis of posterior root tears of the lateral meniscus using arthroscopy as the reference standard. American Journal of Roentgenology 192, 480-486.

Dong, F., Zhang, L., Wang, S., Dong, D., Xu, J., Wu, H., Liu, Y., and Li, M. (2018). The diagnostic accuracy of B-mode ultrasound in detecting meniscal tears: a systematic review and pooled meta-analysis. Medical ultrasonography 20, 164-169.

Gadgil, S., Kakade, A., Bagga, R. P., ZM, and Yadav, M. (2018). MR imaging of meniscal tears. International Journal of Research in Medical Sciences. 6, 195-205.

Lee, M. J., and Chow, K. (2007). Ultrasound of the knee. In "Seminars in musculoskeletal radiology", Vol. 11, pp. 137-148. (C) Thieme Medical Publishers.

Mahdy, N. S., Sakr, H. M., and Allam, A. E. (2018). The Role of Ultrasound in Evaluation of Meniscal Injury. The Egyptian Journal of Hospital Medicine 72, 5490-5494.

Micheo, W., Hernández, L., and Seda, C. (2010). Evaluation, management, rehabilitation, and prevention of anterior cruciate ligament injury: current concepts. $P M \& R$ 2, 935-944.

Mostafa, H. A. M., Abou Elfotuh, A. M., and Alsakka, M. M. (2019). MRI Versus Ultrasound In Diagnosis Of Meniscal Tear In
Knee Joint. The Egyptian Journal of Hospital Medicine 74, 303-309.

Murmu, C., Tiwari, P., and Agrawal, V. K. (2017). Evaluation of medial meniscal injury and anterior cruciate ligament tear by MRI with arthroscopic correlation. International Surgery Journal 4, 589-592.

Najafi, J., Bagheri, S., and Lahiji, F. A. (2006). The value of sonography with micro convex probes in diagnosing meniscal tears compared with arthroscopy. Journal of ultrasound in medicine 25, 593-597.

Nguyen, J. C., De Smet, A. A., Graf, B. K., and Rosas, H. G. (2014). MR imaging-based diagnosis and classification of meniscal tears. Radiographics 34, 981-999.

Nogueira-Barbosa, M. H., Gregio-Junior, E., Lorenzato, M. M., Guermazi, A., Roemer, F. W., Chagas-Neto, F. A., and Crema, M. D. (2015). Ultrasound assessment of medial meniscal extrusion: a validation study using MRI as reference standard. American Journal of Roentgenology 204, 584-588.

Oei, E. H., Nikken, J. J., Verstijnen, A. C., Ginai, A. Z., and Myriam Hunink, M. (2003). MR imaging of the menisci and cruciate ligaments: a systematic review. Radiology 226, 837-848.

Phelan, N., Rowland, P., Galvin, R., and O'Byrne, J. M. (2016). A systematic review and metaanalysis of the diagnostic accuracy of MRI for suspected ACL and meniscal tears of the knee. Knee Surgery, Sports Traumatology, Arthroscopy 24, 1525-1539.

Razek, A., Fouda, N., Elmetwaley, N., and Elbogdady, E. (2009). Sonography of the knee joint. Journal of ultrasound 12, 53-60.

Sally, M. H., and Ali, A. M. (2019). Role of Ultrasound in Assessment of MeniscoLigamentous Injuries of Knee Joint in Comparison with Magnetic Resonance Imaging. The Medical Journal of Cairo University 87, 4571-4577.

Sharma, V., and Grewal, S. (2020). Sonographic evaluation of pathologies of knee joint with MRI correlation. International Journal of Medical Research Professionals 6, 26-33.

Shetty, M. (2013). evaluation of internal derangements of knee joint. Mangalore: (Doctoral dissertation).

Snoeker, B. A., Bakker, E. W., Kegel, C. A., and Lucas, C. (2013). Risk factors for meniscal tears: a systematic review including metaanalysis. journal of orthopaedic \& sports physical therapy 43, 352-367.

[Citation: Omer, M.A., Malik, S.S., Malik, A.A., Anjum, MN., Riaz, A.Z., Ali, R. (2020). Diagnostic accuracy of ultrasound in detecting meniscal tears taking magnetic resonance imaging as gold standard. Biol. Clin. Sci. Res. J., 2020: 40. doi: https://doi.org/10.54112/bcsrj.v2020i1.40] 
Stewart, T. (2010). Tribology of artificial joints. Orthopaedics and Trauma 24, 435-440.

Wang, W., Li, Z., Peng, H.-M., Bian, Y.-Y., Li, Y., Qian, W.-W., Weng, X.-S., Jin, J., Yang, X.Y., and Lin, J. (2019). Accuracy of MRI diagnosis of meniscal tears of the knee: a meta-analysis and systematic review. The journal of knee surgery.

Yaseen, M. K., and Gorial, F. I. (2019). Accuracy of Ultrasound in Comparison to Magnatic Resonance Imaging in Diagnosis of Meniscal Tears. Indian Journal of Public Health Research \& Development 10, 397-400.

Yousif, E. A., Ahmed, B. H., Abdella, A. A., Ali, Q. M., Abdallah, Y. M., Boshara, M. A., Elhaj, A. M., Alshaikh, A. A., Abuidris, D. O., and Eltayeb, A. A. (2014). Knee joint diseases diagnosed by ultrasound and magnetic resonance imaging. Sudan Medical Monitor 9 , 1.

\section{(c) (7) (ㄱ)}

Open Access This article is licensed under a Creative Commons Attribution 4.0 International License, which permits use, sharing, adaptation, distribution and reproduction in any medium or format, as long as you give appropriate credit to the original author(s) and the source, provide a link to the Creative Commons licence, and indicate if changes were made. The images or other third party material in this article are included in the article's Creative Commons licence, unless indicated otherwise in a credit line to the material. If material is not included in the article's Creative Commons licence and your intended use is not permitted by statutory regulation or exceeds the permitted use, you will need to obtain permission directly from the copyright holder. To view a copy of this licence, visit http://creativecommons.org/licen ses/by/4.0/.

(C) The Author(s) 2020

[Citation: Omer, M.A., Malik, S.S., Malik, A.A., Anjum, MN., Riaz, A.Z., Ali, R. (2020). Diagnostic accuracy of ultrasound in detecting meniscal tears taking magnetic resonance imaging as gold standard. Biol. Clin. Sci. Res. J., 2020: 40. doi: https://doi.org/10.54112/bcsrj.v2020i1.40] 